\title{
Control and evaluation of a 7-axis surgical robot for laparoscopy
}

\author{
J. Funda ${ }^{\dagger}$, K. Gruben ${ }^{\dagger}$, B. Eldridge ${ }^{\dagger}$, S. Gomory ${ }^{\dagger}$, R. Taylor ${ }^{\dagger}$ \\ † IBM Thomas J. Watson Research Center, Yorktown Heights, NY 10598 \\ $\ddagger$ Johns Hopkins University, School of Medicine, Baltimore, MD 21205
}

\begin{abstract}
This paper describes the control and ergonomic evaluation of a ceiling mounted (or support frame suspended) 7-axis surgical robot (HISAR) for laparoscopic camera navigation. $A$ key feature of the robot is that it incorporates a passive wrist for natural compliance with the port of entry into the patient. The use of a previously reported constrained Cartesian controller is motivated and demonstrated, and the results of successfully applying this control methodology to the manipulator are presented. The significance of the control strategy is the ease with which control of passive axes, the fulcrum constraint, and the motion inversion effect created by the fulcrum are accommodated. We also report on the results of laboratory evaluations of the arm in terms of its work volume, ergonomic factors, ease of control, and overall design within the context of laparoscopic camera control.
\end{abstract}

\section{Introduction}

A number of key technologies, among them 3D modeling and registration, teleoperation, image processing, sensing and image-based control, have recently reached sufficient levels of maturity and convergence to make their application in medicine, and surgery in particular, practical. This is evidenced by a significant and steadily increasing amount of effort expended in introducing robotic and computer-assisted technology into the operating room by various research groups throughout the world [1][2][3][4][5][6].

One of the medical areas receiving particular attention has been that of minimally invasive surgery, especially laparoscopic surgery. Laparoscopic surgery is characterized by restricted access to the patient's internal organs, loss of direct visualization of the work volume and unnatural hand-eye coordination required of the surgeon to perform these surgeries. To address these problems, a number of computer and robot based systems to assist the laparoscopic surgeon have been recently built and demonstrated in pre-clinical and even clinical settings [7][8][9]. One of these systems ([7]) has been granted FDA approval and is commercially available at the present time.
IBM Research and Johns Hopkins Medical Center are engaged in an ongoing research and development program in image-guided therapy, with the initial focus on laparoscopic surgery. A Laparoscopic Assistant Robot System (LARS) surgical robot was designed and developed at IBM and is currently in pre-clinical in-vivo evaluations at the Johns Hopkins Medical Center[10][11]. LARS is based on a parallel four-bar linkage mechanism with a remote center-ofmotion to accommodate the port of entry into the patient. Concurrently with the evaluation of LARS we decided to explore the ergonomic trade-offs offered by other classes of manipulators. To this end we designed the Hopkins-IBM Surgical Assistant Robot (HISAR), which was built at the prototype level and interfaced to the existing hardware and software architecture of the LARS system.

This paper reports on preliminary evaluations of the kinematic and ergonomic trade-offs offered by HISAR in the context of laparoscopic camera navigation. HISAR is a 7-axis ceiling-mounted (or support frame-suspended) surgical robot for laparoscopic surgery and laparoscopic camera navigation in particular. The arm consists of an active extra-corporeal instrument positioning linkage and a passive two-axis wrist to allow free compliance with the port of entry into the patient. A number of other systems have made use of passive axes to accommodate compliance of the instrument with the port of entry[12][7][8]. In this paper we will motivate and describe the application of constrained Cartesian control to the HISAR arm and report on the results of preliminary investigation of the arm's ergonomic factors. The key contribution of the paper is in demonstrating a method of effectively controlling a surgical robot comprising both active and passive joints. The control formulation is also shown to accommodate additional kinematic and task constraints in an elegant and straightforward manner. This is demonstrated for the case of the HISAR arm, where the control must accommodate not only the hybrid active/passive nature of the mechanism, but must also account for the constraint that all manipulation proceed through a fulcrum and for the resulting mo-

IEEE International Conference

on Robotics and Automation

0-7803-1965-6/95 \$4.00 @ (1995 IEEE 
tion inversion effect.

The remainder of the paper is organized as follows. Section 2 summarizes the general formulation of the Constrained Cartesian control methodology. In Section 3 we describe the geometry and kinematics of the HISAR arm and demonstrate the use of the constrained Cartesian control strategy to control the motion of the robot. This control strategy is then further illustrated (Section 4) on a set of representative view adjustment tasks, typically required for effective navigation of a laparoscope during laparoscopic surgery. Section 5 summarizes the results of the laboratory evaluations of the arm with respect to issues of control and ergonomics. The conclusions are summarized in Section 6.

\section{Constrained Cartesian control}

A number of approaches to controlling redundant mechanisms have been proposed in the literature [13][14][15]. A comparative analysis of these approaches is beyond the scope of this paper. We have recently reported a novel constrained Cartesian control methodology, designed for effective control of both kinematically redundant and deficient robots which must maneuver in constrained workspaces[16]. This section briefly summarizes the control method.

The key problem in controlling kinematically deficient or kinematically redundant mechanisms is determining how best to use the available degrees of freedom (whether too many or too few) of the mechanism in order to accomplish a particular task, while respecting geometric constraints on the work volume, robot mechanism, and the specific task requirements. Let $\left\{f_{1}\right\}, \ldots,\left\{f_{k}\right\}$ denote a set of $k$ Cartesian coordinate frames (task frames), associated with various portions of the robot linkage and its instruments, and which are relevent for the surgical task at hand. Then, any desired displacement of the robot or an instrument can be described in terms of displacements of one or more task frames, along with a (possibly null) set of additional linear constraints, restricting the displacement of each task frame in certain critical DOF to fall within strict user-defined bounds. For situations where a kinematically deficient mechanism is used to perform a task, the formulation allows specification of weights, which can be used to force Cartesian tracking errors in certain DOF to be smaller at the expense of errors in other directions. Having specified the desired Cartesian displacements of a set of task frames, the control problem is to compute the corresponding set of robot's joint displacements $\Delta q$ which will strictly satisfy the specified set of constraints and result in a minimum deviation from the desired motion of the ro- bot and attached instruments in task space. We pose the problem as a quadratic optimization problem of the form:

minimize

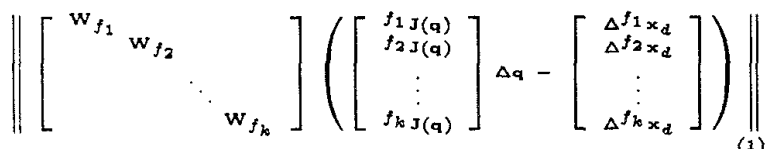

subject to the constraints

$$
\left[\begin{array}{cccc}
\mathrm{H}_{f_{1}} & & & \\
& \mathrm{H}_{f_{2}} & & \\
& & \ddots & \\
& & & \mathrm{H}_{f_{k}}
\end{array}\right]\left[\begin{array}{c}
f_{1} \mathrm{~J}(\mathbf{q}) \\
f_{2} \mathrm{~J}(\mathbf{q}) \\
\vdots \\
f_{k} \mathrm{~J}(\mathbf{q})
\end{array}\right] \Delta \mathbf{q} \geq\left[\begin{array}{c}
\mathrm{h}_{f_{1}} \\
\mathrm{~h}_{f_{2}} \\
\vdots \\
\mathrm{h}_{f_{k}}
\end{array}\right]
$$

The objective function of Eq. (1) is a weighted Euclidean two-norm of the task frame displacement error, where $\Delta^{f} \mathbf{x}_{d}$ denotes the 6-vector of desired positional and orientational displacements of the task frame $\{f\}, \Delta^{f} \mathbf{x}={ }^{f} \mathbf{J}(\mathbf{q}) \Delta \mathbf{q}$ denotes the actual (computed) Cartesian displacement of $\{f\}$, and the diagonal matrix $\mathbf{W}_{f}$ gives the relative importance (weight) of minimizing displacement errors in the individual degrees of freedom of the task frame $\{f\}$. The notation $f \mathbf{J}(\mathbf{q})$ is used to represent the differential Jacobian mapping expressed in the task frame $\{f\}$. Note that a joint space objective function can be specified by designating the $i$-th task frame as the joint space frame and letting ${ }^{f_{i}} \mathbf{J}(\mathbf{q})=\mathbf{I}_{n \times n}, \Delta^{f_{i}} \mathbf{x}_{d}=\Delta \mathbf{q}_{d}$.

Eq. (2) comprises a constraint equation of the form

$$
\mathbf{H}_{f} \cdot{ }^{f} \mathbf{J}(\mathbf{q}) \cdot \Delta \mathbf{q}=\mathbf{H}_{f} \cdot \Delta^{f} \mathbf{x} \geq \mathbf{h}_{f}
$$

for each of the $k$ task frame displacements $\Delta^{f} \mathbf{x}$. In each case $\mathbf{H}_{f}$ denotes the matrix of (constant) constraint coefficients for the task frame $\{f\}$ and $\mathbf{h}_{f}$ represents the vector of specified (constant) constraint bounds. As above, joint space constraints can be specified by letting $f_{i} \mathbf{J}(\mathbf{q})=\mathbf{I}_{n \times n}$.

By consolidating the above matrices Eqs. $(1,2)$ reduce to a linearly constrained quadratic optimization problem of the form

$$
\text { minimize }\|\mathbf{E} \cdot \Delta \mathbf{q}-\mathbf{f}\|, \text { subject to } \mathbf{G} \cdot \Delta \mathbf{q} \geq \mathbf{h} \text { (4) }
$$

where the vector of unknowns to be solved for is the $n$-vector of joint displacements $\Delta \mathbf{q}$. Iterative numerical procedures can be employed to solve this problem in real-time on a personal computer[17][16]. If a solution exists, then the solution to the problem is the $n$-vector of the optimal robot joint displacements $\Delta \mathbf{q}$, which satisfies the specified constraints and minimizes the residual Cartesian motion error as defined by the optimization function. While the formulation could accommodate the requirements and constraints pertaining to the dynamic behavior of the manipulator as 
well, these generally need not be considered for surgical robots, as the motions are deliberately slow for safety reasons.

\section{The HISAR manipulator}

\subsection{HISAR kinematics}

The kinematic structure of HISAR consists of a serial chain of torso $\left(\theta_{1}\right)$, shoulder $\left(\theta_{2}\right)$, elbow $\left(\theta_{3}\right)$, and wrist $\left(\theta_{4}, \theta_{5}\right)$ revolute axes (see Figure 1$)$. Mounted at

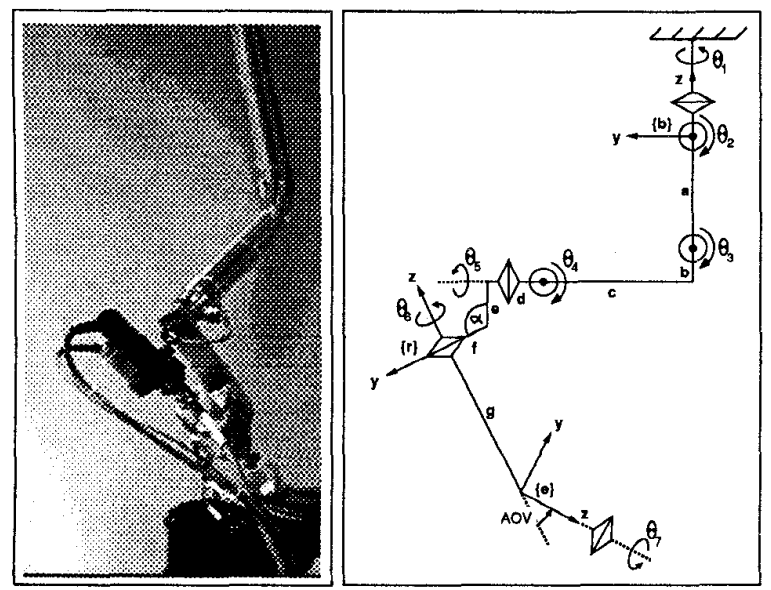

Fig. 1: HISAR robot and its kinematics (at $\theta_{i}=0$ ), where $\{b\},\{r\},\{e\}$ denote the base frame, robot frame, and end-effector frame, respectively, and AOV indicates the angle of view of the telescope.

the wrist is an instrument holder, which allows rotation of the instrument (laparoscope) along its primary axis $\left(\theta_{6}\right)$. In order to allow maintaining the "upright" camera orientation at all times, the rotation of the camera on the eyepiece of the telescope is also motorized $\left(\theta_{7}\right)$. A key feature of the mechanism is that the two wrist axes $\left(\theta_{4}, \theta_{5}\right)$ are passive, i.e., they are encoded but not actuated. This allows the laparoscope to freely comply with the port of entry into the patient, should the patient move during the procedure, thus guaranteeing that the forces exerted by the instrument against the port of entry will be minimal at all times.

Despite the fact that the wrist axes are not actuated, the instrument tip can be positioned accurately as long as the location of the port of entry (fulcrum point) is known exactly and no external forces are acting on the instrument inside the patient. This is because the robot's wrist (which can be precisely positioned anywhere in its workspace) and the instrument tip form an inverted pendulum with the fulcrum point at the port of entry of the instrument into the patient. Thus, under the above assumptions, the position of the instrument tip inside the patient is uniquely determined by the position of the robot's wrist outside the patient. The remaining error (if any) is due to the extent to which the entry portal deviates from an ideal single point pivot.

The kinematic equation for HISAR is given by Eq. (5),

$$
\begin{aligned}
{ }^{b} T_{e}= & R\left(z, \theta_{1}\right) R\left(x, \theta_{2}\right) T(z,-a) R\left(x, \theta_{3}\right) T(z,-b) \\
& T(y, c) R\left(x, \theta_{4}\right) R\left(y, \theta_{5}\right) T(y, d) T(z,-e) \\
& R\left(x,-\alpha^{\prime}\right) T(y, f) R\left(z, \theta_{6}\right)^{r} T_{c} R\left(z, \theta_{7}\right)
\end{aligned}
$$

where $\alpha^{\prime}=\alpha-90^{\circ}$ and ${ }^{r} T_{c}$ is the robot-to-camera coordinate transformation as obtained by the extrinsic camera calibration procedure (e.g., [18]) for the particular laparoscope being used.

\subsection{Control of HISAR}

In this section we will apply the constrained Cartesian control formulation of Section 2 to the task of controlling the HISAR manipulator.

Figure 2 illustrates the geometric arrangement of task frames for laparoscopic camera navigation using the HISAR arm. All view adjustment tasks, such as

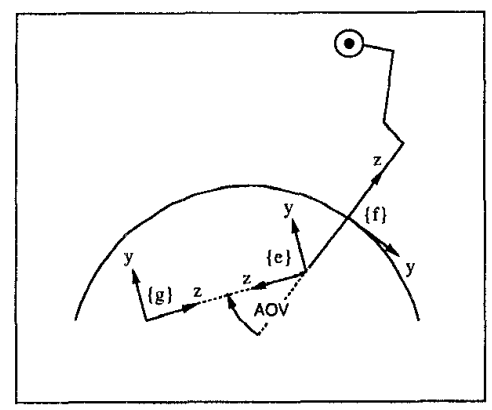

Fig. 2: Task frames for laparoscopic camera navigation. $\{f\},\{e\},\{g\}$ denote the fulcrum frame, endeffector frame, and gaze frame, respectively.

panning, zooming, rotating view, etc. can be specified with respect to one or more of these frames (see Appendix I). Since all camera navigation tasks must be performed through a portal in the patient's abdominal wall and since the HISAR arm has no mechanical remote center of motion to accommodate this constraint, the fulcrum constraint must be enforced by the control software. In particular, in order to be able to correctly control the motion of the instrument tip inside the patient, the control software must be made "aware" of the inverted pendulum effect created by the fixed port of entry of the instrument into the patient. Within the 
framework of the constrained Cartesian motion controller as outlined in Section 2 above, this is easily accomplished by associating a coordinate (task) frame $\{f\}$ with the fulcrum point and adding a corresponding constraint restricting the motion of the fulcrum frame $\{f\}$, i.e.

$$
\left\|\left({ }^{f} \mathbf{J}(\mathbf{q}) \Delta \mathbf{q}\right) \cdot[1,1,0,0,0,0]^{T}\right\| \leq \epsilon_{f}
$$

$\epsilon_{f}$ denotes the computational threshold of the control algorithm and was set to $0.0001 \mathrm{~mm}$ in our experiments. Note that only the $x$ and $y$ components of the translational motion of the fulcrum frame are restricted. This is done to allow the instrument to zoom in and out along the instrument axis.

Note that the fulcrum frame $\{f\}$ is not statically affixed to the kinematic structure of HISAR. Rather, the fulcrum frame moves with the instrument, such that its origin is always coincident with the fulcrum point (port of entry) and its orientation is identical to that of the robot task frame $\{r\}$ (see Figure 1 ). This implies that the control software needs to determine the fulcrum point in order to properly predict the instantaneous location of the fulcrum frame. The more accurately the location of the fulcrum frame is estimated, the more accurately the instrument (camera) tip will track the desired trajectory. Since the orientation of the fulcrum frame $\{f\}$ is the same as that of task frame $\{r\}$, and since the origin of the fulcrum frame lies on the instrument axis, the instantaneous pose of the fulcrum frame is uniquely defined by a scalar parameter $\lambda$, where

$$
{ }^{b} T_{f}={ }^{b} T_{r} T(z,-\lambda) \quad ; \quad \lambda \geq 0
$$

Figure 3 illustrates a method of reestimating the parameter $\lambda$ on-line as the surgical robot is moved about. In the figure $x_{0}$ and $w_{0}$ denote the initial instrument tip and wrist locations, respectively, and $\{f\}_{0}$ corresponds to the actual instantaneous location of the fulcrum frame. Let $\mathbf{x}_{d}$ be the desired Cartesian goal position of the instrument tip and let $\{f\}_{e}$ be the current (incorrect) estimate of the fulcrum's location. Under these assumptions, the target wrist location required to place the instrument tip at $\mathbf{x}_{d}$, will be computed as $\mathbf{w}_{d}$. However, because the fulcrum point is in fact located at $\{f\}_{0}$, the passive wrist joints will conform to this constraint and place the instrument tip at $\mathbf{x}_{a}$, rather than $\mathbf{x}_{d}$, resulting in a Cartesian tool placement error. In order to minimize such errors, the control algorithm continuously reestimates the location of the fulcrum point by computing the intersection ${ }^{1} \mathbf{p}$ of the

\footnotetext{
${ }^{1}$ Since the two lines may not actually intersect, a least squares method is used to compute the 3D point midway along the shortest line segment connecting the two lines.
}

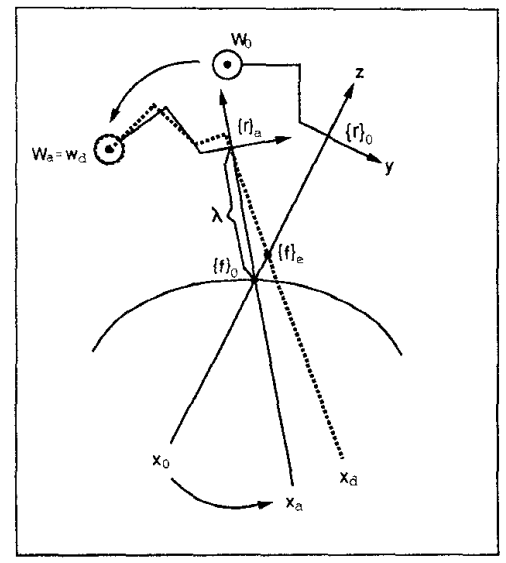

Fig. 3: Real-time tracking of HISAR's fulcrum point.

lines $\overline{\mathbf{r}_{0}, \mathbf{x}_{0}}$ and $\overline{\mathbf{r}_{a}, \mathbf{x}_{a}}$, where all $3 \mathrm{D}$ coordinates have been expressed in a common coordinate frame and $\mathbf{r}_{i}$ denotes the origin of the frame $\{r\}_{i}$. The fulcrum parameter $\lambda$ can then be computed as

$$
\lambda=\left\|\mathbf{p}-\mathbf{r}_{a}\right\|
$$

The coordinates of $\mathbf{r}$ and $\mathbf{x}$ are by-products of the computation of the forward kinematics and can thus be updated at no extra cost at each control cycle. In order to improve the numerical stability of the fulcrum point estimation (3D line intersection), the fulcrum point is recomputed only after the wrist has moved at least $5 \mathrm{~mm}$ in the $x y$ plane of the fulcrum frame, i.e., orthogonally to the current instrument axis.

\section{Experimental results}

Four representative laparoscopic view adjustment tasks have been chosen to illustrate the behavior of the HISAR arm employing the control strategy outlined above. The detailed formulations of the four control tasks in terms of the constrained Cartesian control formalism are given in Appendix I. Figure 4 illustrates the tracking performance of the HISAR arm for the four control tasks. A $30^{\circ}$ laparoscope was used in all experiments reported below.

For each of the tasks the desired (thin lines) and actual computed (thick lines) trajectories of the gaze frame $\{g\}$ are shown, as well as the translational motion of the fulcrum frame (dashed lines). Figure 4 shows that the translational displacement of the fulcrum point (e.g., origin of fulcrum frame) indeed conforms to the fulcrum constraint (Eq. (6)) and is effectively zero in the $x$ and $y$ directions, as required. The $z$ direction of the fulcrum point motion is not constrained and is in general non-zero. Also evident from 


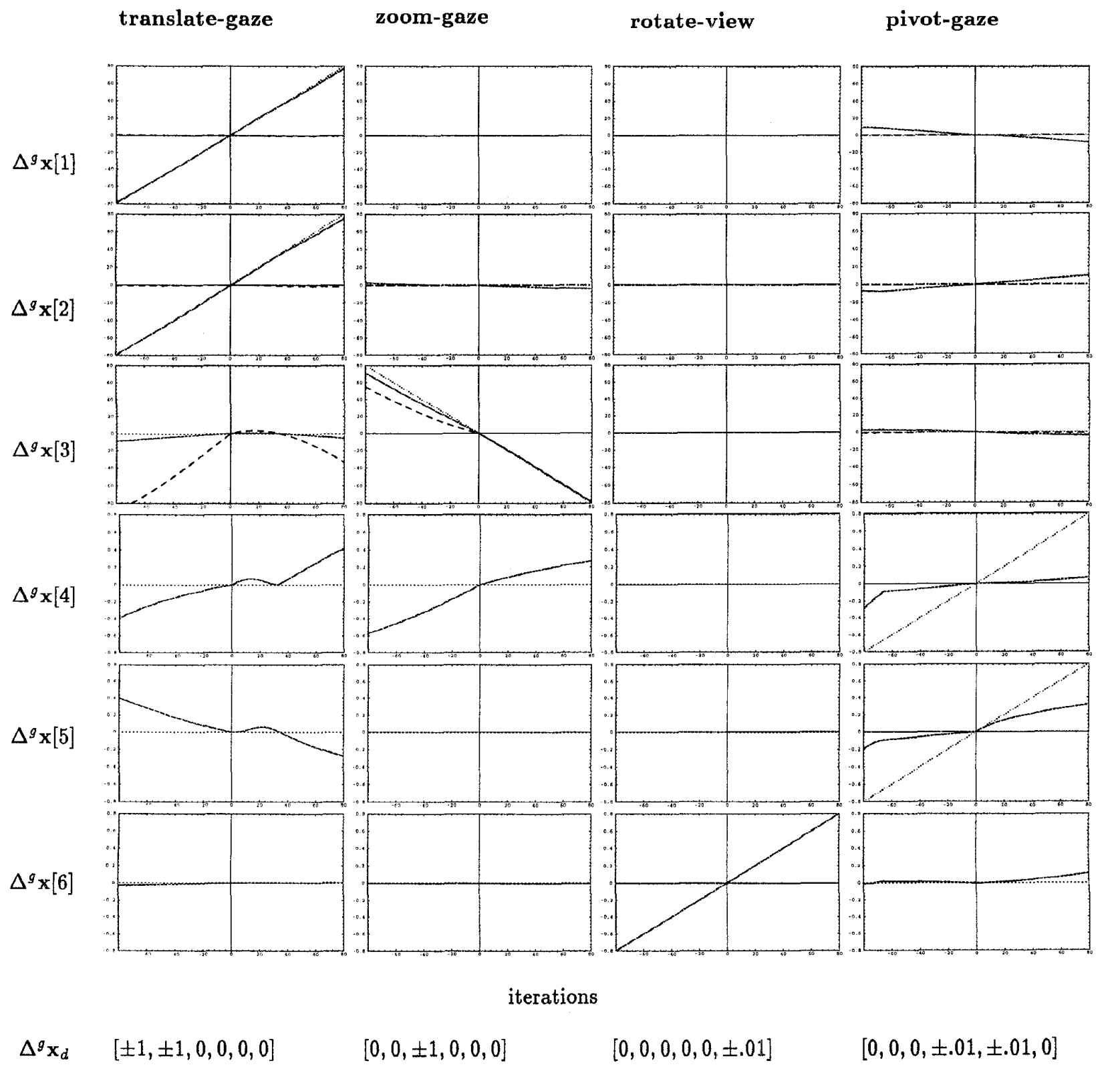

Fig. 4: Experimental results of the four view control tasks using the HISAR surgical robot. Actual computed gaze-frame (thick lines) trajectories are shown against their commanded trajectories (thin lines). The motion of the fulcrum point is shown in dashed lines. $\Delta^{g} \mathbf{x}_{d}$ gives the desired incremental motion $(\mathrm{mm}$, rad) at each step of the iteration. 
the figure is the fact that the HISAR arm is kinematically deficient for three of the four control tasks. This is to be expected since an unarticulated instrument operating through a fulcrum only has 4 degrees-offreedom (DOF) of motion (three rotational, one translational) about the fulcrum. Of the four tasks, only rotate-view task can be executed without error as this task corresponds to the rotation of the camera on the eyepiece of the telescope. The constrained Cartesian controller is thus able to find an exact solution and the trajectory tracking is exact. On the other hand, the plotted trajectories of the gaze-frame (solid lines) in all other modes exhibit varying amounts of tracking error in different Cartesian degrees of freedom, as expected. For instance, in translate-gaze mode, very close tracking of the positional gaze-frame trajectories comes at the expense of rotational errors in $x$ and $y$ directions. Similarly, zoom-gaze mode provides good tracking of the key translational DOF but incurs a substantial rotational error about the $x$ axis of the gaze frame. Predictably, the poorest tracking behavior is exhibited by pivot-gaze task, as the necessary degrees of freedom to accomplish the pivoting task are effectively unavailable to the mechanism.

In general, for 6-DOF view adjustment tasks, tracking errors are unavoidable as the HISAR mechanism is kinematically deficient for the task. However, the importance of the above trajectories is that they optimally approximate the desired trajectories, subject to the constraints imposed by the robot, the work volume, and the task. The trade-offs in the tracking errors in the different DOF reflect the particular choice of optimization weights given in Appendix I. Other choices of weights could be used to force errors in certain DOF to become smaller at the expense of errors in other DOF.

\section{Discussion}

We have successfully implemented and demonstrated the above control methodology on the prototype HISAR arm. The laboratory experimental setup consisted of a laparoscopic simulator with rubber portals for instruments, and the prototype HISAR arm suspended from a frame above the simulator (Figure 5). Both $0^{\circ}$ and $30^{\circ}$ telescopes were used in the laboratory experiments.

During the course of experimentation we found that the ceiling-mounted (or frame-suspended) mechanical structure of HISAR was relatively unintrusive into the surgical workspace and that the range of motion of the mechanism was sufficient to allow the operator to position the instrument in any configuration which would have been reachable manually. The only signif-

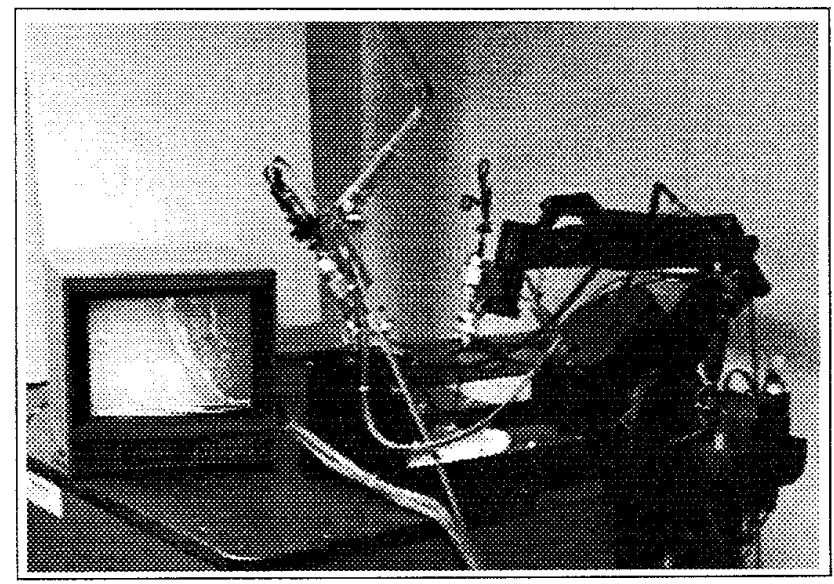

Fig. 5: The experimental setup for HISAR evaluations. The LARS surgical robot (to the right of HISAR) is holding a surgical instrument.

icant kinematic deficiency of HISAR is that the extracorporeal linkage, which is used to position the robot's wrist (and thus the instrument carrier) exhibits a kinematic singularity when the wrist lies along (or close to) the axis of the "torso" joint $\left(\theta_{1}\right)$, i.e., when

$$
a \cdot \sin \left(\theta_{2}\right)+l \cdot \cos (\beta)=0
$$

where

$$
l=\sqrt{b^{2}+c^{2}} \quad ; \quad \beta=\theta_{2}-\theta_{3}+\arctan (b / c)
$$

as illustrated in Figure 6. In this singular configuration

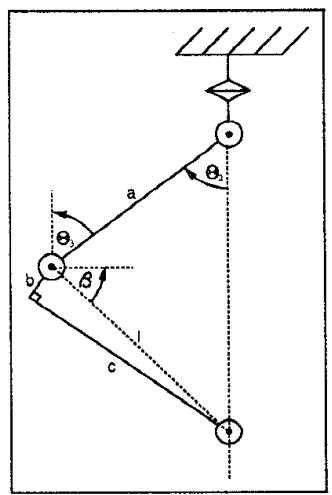

Fig. 6: HISAR's kinematic singularity.

wrist motions in the directions perpendicular to the plane of the linkage are not possible. Unfortunately, this singularity is located in the central and potentially often traversed portion of the mechanism's workspace and is thus very undesirable. A possible solution would 
be a modification of the "upper" arm of the linkage to include a torsional degree of freedom along the axis of the link, yielding a redundant 4 DOF extra-corporeal positioning mechanism, which could then be controlled so as to avoid singular configurations.

Real-time fulcrum tracking was successfully demonstrated, but we found that the computation of the estimated fulcrum point location was subject to small but significant non-systematic error due to the deviation of the physical fulcrum from the ideal point pivot (assumed by the mathematics). This was exacerbated by the buckling of the rubber portals used in our laboratory simulator. The proper way to address this problem would be to use a more sophisticated and more robust on-line fulcrum point estimation algorithm. We have found, however, that this was not necessary as the error in estimation of the instantaneous fulcrum point (and the corresponding error in the interpretation of the commanded joystick input) did not affect the subjective feel and qualitative response of the system for the simple task of positioning and navigating a laparoscopic camera.

In general, we found that the control of the system is good and sufficiently accurate for the task of laparoscope navigation so long as no external forces are acting on the instrument inside the patient. When the instrument comes into contact with the tissue and its motion is thus restricted, subsequent motion of the instrument is unpredictable, as passive axes tend to comply with any and all existing constraints on the motion of the instrument. Thus, while the use of passive axes offers the inherent safety of the instrument complying with the location of the entry portal, it makes the system unsuitable for applications where precise positioning of an instrument which forcibly interacts with tissues is necessary.

\section{Conclusion}

An effective control methodology for control of a surgical robot comprising both active and passive joints has been demonstrated on a 7-axis robot for laparoscopic camera navigation. The constrained Cartesian control formulation has been shown to provide a powerful and convenient language for describing arbitrary goals in the task space of the manipulator, subject to any number of absolute constraints on the allowable motion envelope. These constraints can be dictated by the robot mechanism itself (e.g., joint limits), work space limitations, or the nature of the task. The fact that the HISAR arm consists of a combination of active and passive axes and that the compliance of the passive axes with the port of entry creates a motion inversion effect was shown to be easily accommodated within the framework of the constrained Cartesian control. We have successfully demonstrated the control of the task-deficient HISAR arm on a number of typical tasks within the domain of laparoscopic camera navigation. The results confirm the correctness, effectiveness and flexibility of the control strategy. When the DOF of motion to accomplish a particular task are available to the mechanism, the desired motion trajectories are tracked exactly, as expected. On the other hand, when the mechanism is kinematically deficient for the task, the controller makes mathematically optimal and intuitively predictable trade-offs between tracking errors in the various DOF, subject to user specified penalty weights. Finally, we have shown that the HISAR mechanism is ergonomically well suited for the surgical environment and can be successfully used for laparoscopic camera manipulation. However, the existence of a centrally located kinematic singularity and the fact that instrument motion is not predictable in the presence of external forces make it unsuitable for applications such as biopsies or therapy injections, where precise control of an instrument is necessary while the instrument is forcibly interacting with the tissue.

\section{Appendix I Formulation of the four view control tasks}

The four control tasks discussed in Section 4 were implemented as the following constrained Cartesian motions. See Section 2 for a description of the control methodology.

\begin{tabular}{|c|c|l|}
\hline frm & $\mathbf{c} / \circ$ & translate-gaze \\
\hline$\{f\}$ & $\mathrm{c}$ & $\left\|\left(\Delta^{f} \mathbf{x}\right) \cdot[1,1,0,0,0,0]^{T^{T}}\right\| \leq 0.0001$ \\
\hline$\{c\}$ & $\circ$ & - \\
\hline$\{g\}$ & $\circ$ & $\left\|\mathbf{W}_{g}\left(\Delta^{g} \mathbf{x}-\Delta^{g} \mathbf{x}_{d}\right)\right\|, \mathbf{w}_{g}=[H H H L L H]$ \\
\hline$\{j\}$ & $\mathbf{c}$ & $\mathbf{q}-\mathbf{q} \leq \Delta \mathbf{q} \leq \overline{\mathbf{q}}-\mathbf{q}$ \\
& $\circ$ & $\left\|\mathbf{W}_{j} \Delta \mathbf{q}\right\|, \mathbf{w}_{j}=[L \ldots L]$ \\
\hline
\end{tabular}

\begin{tabular}{|c|c|l|}
\hline frm & $c / o$ & zoom-gaze \\
\hline$\{f\}$ & $\mathbf{c}$ & $\left\|\left(\Delta^{f} \mathbf{x}\right) \cdot[1,1,0,0,0,0]^{T}\right\| \leq 0.0001$ \\
\hline$\{c\}$ & $\mathrm{o}$ & $\left\|\mathbf{W}_{c}\left(\Delta^{c} \mathbf{x}-\Delta^{c} \mathbf{x}_{d}\right)\right\|, \mathbf{w}_{c}=[L L H L L H]$ \\
\hline$\{g\}$ & $\mathbf{o}$ & $\left\|\mathbf{W}_{g}\left(\Delta^{g} \mathbf{x}+\Delta^{c} \mathbf{x}_{d}\right)\right\|, \mathbf{w}_{g}=[L L H L L H]$ \\
\hline$\{j\}$ & $\mathbf{c}$ & $\mathbf{q}-\mathbf{q} \leq \Delta \mathbf{q} \leq \overline{\mathbf{q}}-\mathbf{q}$ \\
& $\circ$ & $\left\|\mathbf{W}_{j} \Delta \mathbf{q}\right\|, \mathbf{w}_{j}=[L \ldots L]$ \\
\hline
\end{tabular}

\begin{tabular}{|c|c|l|}
\hline frm & c/o & rotate-view, pivot-gaze \\
\hline$\{f\}$ & $\mathbf{c}$ & $\left\|\left(\Delta^{f} \mathbf{x}\right) \cdot[1,1,0,0,0,0]^{T^{\prime}}\right\| \leq 0.0001$ \\
\hline$\{c\}$ & - & - \\
\hline$\{g\}$ & 0 & $\left\|\mathbf{W}_{g}\left(\Delta^{g} \mathbf{x}-\Delta^{g} \mathbf{x}_{d}\right)\right\|, \mathbf{w}_{g}=[L L L H H H]$ \\
\hline$\{j\}$ & $\mathbf{c}$ & $\mathbf{q}-\mathbf{q} \leq \Delta \mathbf{q} \leq \overline{\mathbf{q}}-\mathbf{q}$ \\
& $\circ$ & $\left\|\mathbf{W}_{j} \Delta \mathbf{q}\right\|, \mathbf{w}_{j}=[L \ldots L]$ \\
\hline
\end{tabular}


For each of the four control tasks, the tables above list the set of objectives (o) and constraints (c) with respect to the various task frames (frm), which are relevant to the task. $\{f\},\{c\},\{g\},\{j\}$ denote the fulcrum frame, camera frame, gaze frame, and the "joint space frame", respectively. The diagonal weight matrices $\mathbf{W}_{f}$ are given as vectors $\mathbf{w}_{f}$ of diagonal elements, where $\mathrm{H}, \mathrm{M}$, and $\mathrm{L}$ denote high, medium, and low weight, respectively. Note that for the first three tasks the desired motion is given in terms of the motion of the gaze frame, whereas in the case of zoom-gaze, the desired motion is more naturally expressed with respect to the camera frame. Also note that in the case of zoom-gaze task, the displacement of the gaze frame is the negative of the camera frame displacement $\Delta^{c} \mathrm{x}=[0,0, d z, 0,0,0]^{T}$, since the $z$-axes of the camera and gaze frames are colinear but opposite. Since the HISAR arm is kinematically deficient for the general $6 \mathrm{DOF}$ view adjustment, no absolute constraints on the accuracy of the gaze-frame trajectory tracking have been specified. Finally, all control tasks include a joint space constraint (making sure that joint limits are respected) and a joint space objective (which attempts to minimize the total joint motion).

\section{References}

[1] B. A. Kall, P. J. Kelly, and S. J. Goerss. Interactive stereotactic surgical system for the removal of intracranial tumors utilizing a $\mathrm{CO} 2$ laser and $\mathrm{CT}$ derived database. IEEE Transactions on Biomedical Engineering, pages 112-116, February 1985.

[2] S. Lavallee. A new system for computer-assisted neurosurgery. In Proc. of $11^{\prime}$ th IEEE Engineering in Medicine and Biology Conference, pages 926-927, November 1989 .

[3] S. Charles, R. E. Williams, and B. Hammel. Design of a surgeon-machine interface for teleoperated microsurgery. In Proceedings of EMBS '89, pages 883-884, 1989.

[4] B. L. Davies, R. D. Hibberd, A. Timoney, and J. Wickham. A surgeon robot for prostatectomies. In Proc. of the Fifth International Conference on Advanced Robotics, pages 871-875, June 1991.

[5] H. Paul, B. Mittlestadt, W. Bargar, B. Musits, R. Taylor, P. Kazanzides, J. Zuhars, B. Williamson, and W. Hanson. A surgical robot for total hip replacement surgery. In Proceedings of the IEEE International Conference on Robotics and Automation, pages 606-611, Nice, France, May 1992.

[6] J. W. Hill, P. S. Green, J. F. Jensen, Y. Gorfu, and Ajit S. Shah. Telepresence surgery demonstration system. In Proceedings of the IEEE International Conference on Robotics and Automation, pages 2302-2307, San Diego, CA, May 1994.
[7] Y. Wang and J. Sackier, MD FACS. Robotically enhanced surgery. In Proc of Medicine meets virtual reality II, San Diego, January 1994.

[8] R. Hurteau, S. DeSantis, E. Begin, and M. Garner. Laparoscopic surgery assisted by a robotic cameraman: Concept and experimental results. In Proceedings of the IEEE International Conference on Robotics and Automation, pages 2286-2289, San Diego, CA, May 1994.

[9] J. B. Petelin. Computer assisted surgical instrument control. In Proc. of Medicine Meets Virtual Reality II., pages 170-173, San Diego, CA, January 1994.

[10] R. Taylor, J. Funda, B. Eldridge, K. Gruben, D. LaRose, S. Gomory, and M. Talamini, MD. A telerobotic assistant for laparoscopic surgery. To appear in IEEE EMBS magazine, special issue on Robotics in Surgery, late 1994.

[11] J. Funda, R. Taylor, S. Gomory, B. Eldridge, K. Gruben, and M. Talamini, MD. An experimental user interface for an interactive surgical robot. In Proceedings of the 1st Int'l Symposium of Medical Robotics and Computer Assisted Surgery, Pittsburgh, PA, September 1994.

[12] J. A. McEwen. Solo surgery with automated positioning platforms. In Proc. of NSF Workshop on Computer Assisted Surgery, pages D89-D98, Washington, D.C., 1993.

[13] D. E. Whitney. Resolved motion rate control of manipulators and human prostheses. IEEE Transactions on Man-Machine Systems, 10:47-53, 1969.

[14] J. Baillieul. Kinematic programming alternatives for redundant manipulators. In Proceedings of the IEEE International Conference on Robotics and Automation, pages 722-728, St. Louis, MO, March 1985.

[15] Y. Nakamura and H. Hanafusa. Robotics Research: The Second International Symposium, chapter Task priority based redundancy control of robot manipulators. MIT Press, 1985.

[16] J. Funda, R. Taylor, K. Gruben, and D. LaRose. Optimal motion control of teleoperated surgical robots. In Proc. of the 1999 SPIE Int'l Symp. on Optical Tools for Manufacturing and Advanced Automation, Boston, MA, September 1993.

[17] C. Lawson and R. Hanson. Solving Least Squares Problems. Prentice Hall, 1974.

[18] Roger Y. Tsai. A versatile camera calibration technique for high-accuracy $3 \mathrm{D}$ machine vision metrology using off-the-shelf TV cameras and lenses. IEEE Transactions on Robotics and Automation, RA3(4):323-344, 1987. 